\title{
No infectious SARS-CoV-2 in breast milk from a cohort of 110 lactating women
}

\author{
Paul Krogstad ${ }^{1,2}$, Deisy Contreras ${ }^{1}$, Hwee $\mathrm{Ng}^{1}$, Nicole Tobin ${ }^{1}$, Christina D. Chambers ${ }^{3,4}$, Kerri Bertrand ${ }^{3,4}$, Lars Bode ${ }^{3,5}$ and \\ Grace M. Aldrovandi ${ }^{1}$
}

(c) The Author(s) 2021

\begin{abstract}
BACKGROUND: Genomic RNA of severe acute respiratory syndrome-associated coronavirus type 2 (SARS-CoV-2) has been detected in the breast milk of lactating women, but its pathological significance has remained uncertain due to the small size of prior studies. METHODS: Breast milk from 110 lactating women was analyzed by reverse transcription-polymerase chain reaction (285 samples) and viral culture (160 samples). Those containing SARS-CoV-2 viral RNA (vRNA) were examined for the presence of subgenomic RNA (sgRNA), a putative marker of infectivity.

RESULTS: Sixty-five women had a positive SARS-CoV-2 diagnostic test, 9 had symptoms but negative diagnostic tests, and 36 symptomatic women were not tested. SARS-CoV-2 vRNA was detected in the milk of 7 (6\%) women with either a confirmed infection or symptomatic illness, including 6 of 65 (9\%) women with a positive SARS-CoV-2 diagnostic test. Infectious virus was not detected in any culture and none had detectable sgRNA. In control experiments, infectious SARS-CoV-2 could be cultured after addition to breastmilk despite several freeze-thaw cycles, as it occurs in the storage and usage of human milk.

CONCLUSIONS: SARS-CoV-2 RNA can be found infrequently in the breastmilk after recent infection, but we found no evidence that breastmilk contains an infectious virus or that breastfeeding represents a risk factor for transmission of infection to infants.
\end{abstract}

Pediatric Research (2022) 92:1140-1145; https://doi.org/10.1038/s41390-021-01902-y

\section{IMPACT:}

- This article goes beyond prior small studies to provide evidence that infectious SARS-CoV-2 is not present in the milk of lactating women with recent infection, even when SARS-CoV-2 RNA is detected.

- Recent SARS-CoV-2 infection or detection of its RNA in human milk is not a contraindication to breastfeeding.

\section{INTRODUCTION}

To date, the coronavirus disease 2019 (COVID-19) pandemic has infected nearly 170 million people globally and over 33 million people in the United States. ${ }^{1}$ COVID-19 cases in infants represent about $50 \%$ of the total reported in young children globally, ${ }^{2}$ and infections of infants represent approximately $1.9 \%$ of all cases in the United States. ${ }^{3}$ Most newborns and infants fare well, but severe disease and death have been reported in newborns, infants, and young children. ${ }^{2}$ In addition, the multisystem inflammatory syndrome in children can occur even after apparent resolution of infection and is disproportionately affecting Black and Hispanic/Latino children. ${ }^{4}$ Understandably, there has been great concern about the potential consequences of severe acute respiratory syndrome-associated coronavirus type 2 (SARS-CoV-2) transmission to infants by postnatal exposures, including breastfeeding. There is limited but increasing evidence that breast milk is not a source of SARS-CoV-2 infection to infants who are breastfed by mothers who have evidence of recent infection., Therefore, the US Centers for Disease Control and Prevention, the
American Academy of Pediatrics, and the World Health Organization advise that mothers who are infected with SARS-CoV-2 may choose to initiate or to continue breastfeeding an infant or toddler with appropriate protections to prevent transmission of the virus through respiratory droplets. ${ }^{7,8}$

We previously presented initial results from a study of 64 breast milk samples from 18 women with recent SARS-CoV-2 infection. ${ }^{9} \mathrm{~A}$ sample from one symptomatic woman was found to contain SARS-CoV-2 viral RNA (vRNA), but replication-competent virus was not detected in viral culture. In addition, we used viral culture methods to demonstrate that the conditions of Holder pasteurization inactivated SARS-CoV-2, a finding subsequently confirmed by others. ${ }^{10-12}$ To better examine the frequency and state of SARSCoV-2 in the breast milk of women with recently documented infection, we present results from a larger observational cohort study. We also examined the vRNA found in a reverse transcription-polymerase chain reaction (RT-PCR)-positive samples for the presence of SARS-CoV-2 subgenomic transcripts, a proposed marker of viral infectivity. ${ }^{13-15}$ In addition, we examined

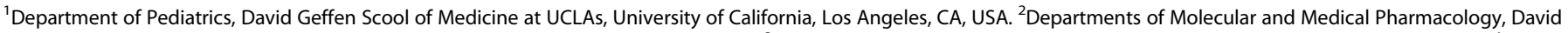

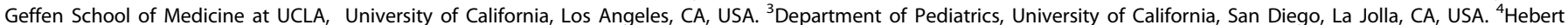

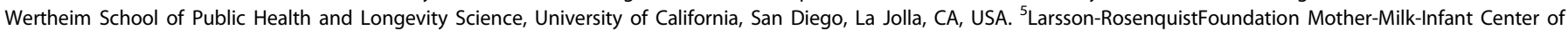
Research Excellence (MOMI CORE), University of California, San Diego, La Jolla, CA, USA. ${ }^{\circledR}$ email: pkrogstad@mednet.ucla.edu

Received: 31 May 2021 Revised: 30 August 2021 Accepted: 17 September 2021

Published online: 19 January 2022 
the impact of breast milk on SARS-CoV-2 thermal stability to investigate the apparent discordance between viral culture and vRNA detection.

\section{MATERIALS AND METHODS}

\section{Participants and breast milk specimens}

As previously described, ${ }^{9}$ breast milk samples and clinical information were obtained from women participating in the Mommy's Milk Human Milk Biorepository at the University of California, San Diego (IRB\#130658). Between March 2020 and September 2020, we enrolled women residing in the United States who were symptomatic but not tested, symptomatic but with negative SARS-CoV-2 testing by RT-PCR, women exposed to an infected person, or those who had a confirmed SARS-CoV-2 infection by RT-PCR. Information about demographics, health history, illness and exposure dates, symptoms, and SARS-CoV-2 test results were collected by participant interview via telephone. Participants self-collected breast milk samples using a provided collection kit including instructions for expressing and storing their samples. The instructions provided specific instructions for hand washing before and after milk expression. Participants who had recovered from their illness at the time of the study interview were asked to ship any frozen samples previously collected at the peak of their symptoms in addition to a fresh milk sample. Fresh samples were shipped on ice within $24 \mathrm{~h}$ of collection to the Biorepository and stored at $-80^{\circ} \mathrm{C}$ prior to shipment on dry ice to the University of California, Los Angeles. This report includes samples from 18 women described in our previous report. ${ }^{9}$

\section{Virologic methods}

The PCR-based methods used to detect SARS-CoV-2 RNA in skim milk and the culture techniques to detect replication-competent SARS-CoV-2 in whole breast milk have been previously described. ${ }^{9}$ The concentration of replication-competent virus in SARS-CoV-2 viral stocks was determined by limiting dilution culture in Vero-E6 (Cercopithecus aethiops-derived epithelial kidney) cells, calculated using the Spearman-Karber method, and expressed as the median tissue culture infectious doses/mL $\left(\mathrm{TCID}_{50} /\right.$ $\mathrm{mL}$ ). RT-PCR analysis to detect subgenomic SARS-CoV-2 RNA (a proposed marker of viral replication ${ }^{13-15}$ ) was performed using oligonucleotide PCR primers WHSA-00025F and WHSA29925R ${ }^{13}$ and a Power Syber Green RNA to Ct 1-Step Kit (Thermo Fisher Scientific), following the manufacturer's instructions.

\section{Thermal stability of SARS-CoV-2}

We previously reported that SARS-CoV-2 could not be grown from a whole breast milk specimen that was shown to contain a high concentration of

Table 1. Characteristics of 66 women with confirmed SARS-CoV-2 infection ${ }^{\mathrm{a}}$.

\begin{tabular}{|c|c|c|c|}
\hline & $\begin{array}{l}\text { No detectable SARS-CoV-2 in breast milk } \\
\text { samples, } n(\%), N=59\end{array}$ & $\begin{array}{l}\text { Detectable SARS-CoV-2 in breast milk } \\
\text { samples, } n(\%), N=7\end{array}$ & $P$ value \\
\hline \multicolumn{3}{|l|}{ Race } & 1.000 \\
\hline Black & $1(1.75)$ & $0(0.00)$ & \\
\hline Asian & $5(8.77)$ & $0(0.00)$ & \\
\hline \multicolumn{3}{|l|}{ Ethnicity } & 0.528 \\
\hline Hispanic & $5(8.77)$ & $1(14.29)$ & \\
\hline Non-Hispanic & $52(91.23)$ & $6(85.71)$ & \\
\hline \multicolumn{3}{|l|}{ US region of residence } & 0.019 \\
\hline North-East & $21(35.59)$ & $1(14.29)$ & \\
\hline $\mathrm{BMI} \geq 30$ & $20(35.08)$ & $0(0.00)$ & 0.096 \\
\hline Underlying health condition ${ }^{\mathrm{b}}$ & $15(26.32)$ & $2(28.57)$ & 1.000 \\
\hline Symptomatic & $53(89.83)$ & $6(100.00)$ & 1.000 \\
\hline Hospitalized & $6(10.71)$ & $0(0.00)$ & 1.000 \\
\hline Treatment received: Remdesivir & $3(5.26)$ & $0(0.00)$ & 1.000 \\
\hline Treatment received: ECMO & $2(3.51)$ & $0(0.00)$ & 1.000 \\
\hline \multicolumn{3}{|l|}{ Source of infection } & 0.517 \\
\hline Household & $17(29.31)$ & $3(42.86)$ & \\
\hline Community & $11(18.97)$ & $2(28.57)$ & \\
\hline Occupational & $18(31.03)$ & $2(28.57)$ & \\
\hline
\end{tabular}

${ }^{a}$ Sixty-five who tested positive by RT-PCR of the nasopharyngeal specimen and 1 without diagnostic testing whose breast milk sample was positive by RT-PCR. bUnderlying medical conditions included the following: asthma, diabetes type II, heart defect, hypertension, hypothyroidism, irritable bowel disease, kidney defect, obesity, and "tachycardia". 


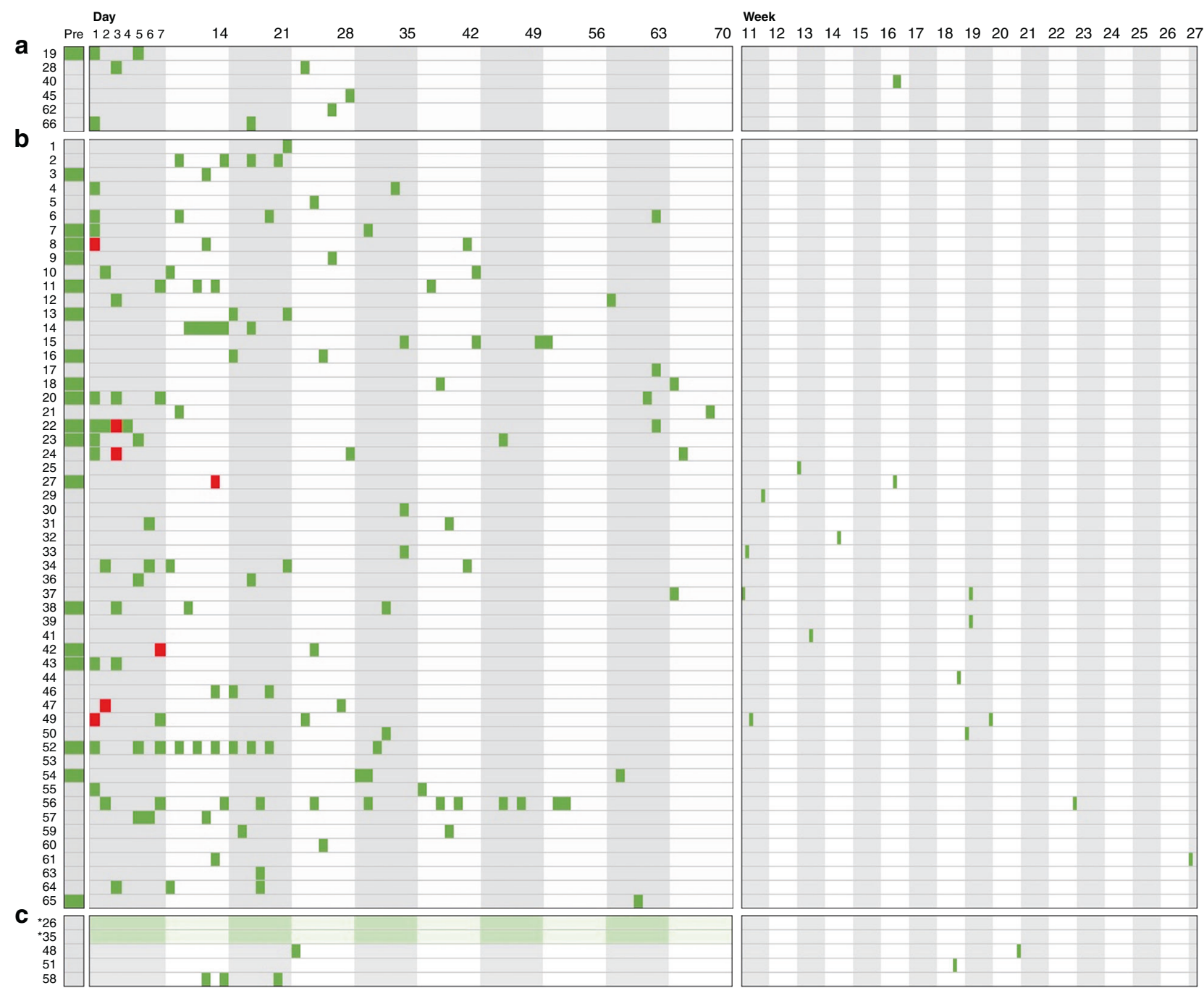

Fig. 1 Sample collection timing by day/week from the onset of symptoms or hospitalization for 65 women who tested positive for SARSCoV-2 infection and 1 woman who was symptomatic but untested. Panel a displays the timing of breast milk samples collected from women who lacked any symptoms of COVID-19 (SARS-CoV-2 infection). Panel b displays the timing of breast milk samples collected by symptomatic women who were not hospitalized. Panel c displays are those who were symptomatic and hospitalized. Each colored rectangle demonstrates the time of collection of breast milk specimens in relation to their onset of SARS-CoV-2 symptoms or for those women who were asymptomatic, in relation to their positive test date. Red squares indicate specimens in which the RNA of SARS-CoV-2 was detected by RT-PCR. Asterisks indicate the two women (\#26 and \#35) who required ECMO support; the date of collection is not precisely known and green shading is therefore used to indicate the period during which the breast milk was collected.

SARS-CoV-2 RNA. ${ }^{9}$ In that report, we also noted that the temperature conditions of Holder pasteurization rapidly inactivated live virus, even if diluted ten-fold in breast milk from healthy donors. However, it remained possible that we were unable to grow SARS-CoV-2 due to the presence of antimicrobial factors in breast milk that could reduce viral infectivity during the cycles of freezing and gradual low-temperature thawing that commonly occur with storage and preparation of breast milk. To examine the thermal stability of the virus during these steps, we added a small amount $\left(100 \mathrm{TCID}_{50}\right)$ of SARS-CoV-2 (USA-WA1/2020) to four aliquots each of whole breast milk from two different healthy women. One aliquot from each set was then held at $4{ }^{\circ} \mathrm{C}$, and three others were frozen again at -80 ${ }^{\circ} \mathrm{C}$. These aliquots were slowly thawed to room temperature (approximately $20^{\circ} \mathrm{C}$ ). Two aliquots were frozen again to $-80^{\circ} \mathrm{C}$, and one was held at $4{ }^{\circ} \mathrm{C}$. This process was repeated, yielding samples that had undergone two freeze-thaw cycles. Following a third freeze and thaw for aliquots from both women, all samples were stored at $4^{\circ} \mathrm{C}$ for 3 days. Using previously described methods, ${ }^{9}$ we inoculated viral cultures with this spiked milk and examined them for cytopathic effect (normally easily identified by 4 days of culture). After 4 days of culture, RNA was extracted from the culture supernatants and RT-PCR ${ }^{9}$ was used to detect SARS-CoV2 genomic RNA.

\section{Statistical analysis}

Maternal and infant characteristics were compared between the group of mothers with confirmed infection for whom no milk samples were positive and those with either confirmed infection or who were symptomatic and who had at least one milk sample that was positive for vRNA. Comparisons for continuous variables were made using Wilcoxon's rank-sum test. Categorical variables were compared using Fisher's exact test. Missing values were excluded. SPSS version 25 was used for analyses, and Prism version 8.4.3 (GraphPad) was used for figure presentation.

\section{RESULTS}

Detection of SARS-CoV-2 RNA in the breast milk of women Breast milk samples were available from 110 women: 36 women with SARS-CoV-2 symptoms, but no diagnostic test, 9 women with symptoms but a negative nasal or nasopharyngeal SARS-CoV-2 RTPCR test, and 65 study participants with confirmed SARS-CoV-2 using a nasal or nasopharyngeal RT-PCR test (including 18 previously described women ${ }^{9}$ ). A total of 336 breast milk samples were submitted by these participants (median of 2.5 samples of 
Table 2. Summary of virologic data from breast milk samples with detectable SARS-CoV-2 RNA.

\begin{tabular}{|c|c|c|c|c|c|c|}
\hline Participant & $\begin{array}{l}\text { Number of } \\
\text { symptoms }{ }^{a}\end{array}$ & $\begin{array}{l}\text { Number of days } \\
\text { symptomatic }\end{array}$ & $\begin{array}{l}\text { Symptomatic at time of } \\
\text { sample collection }\end{array}$ & $\begin{array}{l}\text { SARS-CoV-2 RNA in } \\
\text { milk samples (copies/ } \\
\mathrm{mL} \text { ) }\end{array}$ & sgRNA & Viral culture \\
\hline $8^{b}$ & 9 & 18 & Yes & 25,100 & Negative & Negative \\
\hline 24 & 16 & 65 & Yes & 3230 & Negative & Negative \\
\hline $27^{c}$ & 9 & 13 & Yes & 10,000 & Negative & Negative \\
\hline 49 & 7 & 7 & Yes & 150 & Negative & Negative \\
\hline
\end{tabular}

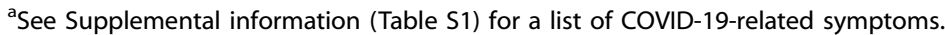

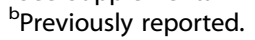

'Woman was symptomatic but not tested.

breast milk/woman (range 1-13). Out of 336 samples, 285 breast milk samples (85\%) were available and analyzed for SARS-CoV-2 vRNA (six unsuccessful PCR assays). A total of 160 samples were cultured for the virus: 118 from 50 of the women with positive SARS-CoV-2 nasopharygeal PCR tests and 42 from the two groups of symptomatic women. All subsequent descriptors presented below are restricted to the 65 women who had a confirmed diagnosis of SARS-CoV-2 and 1 asymptomatic woman whose milk sample was positive for vRNA, but who was not tested for SARSCoV-2. We will refer to this group as the 66 women with confirmed SARS-CoV-2 infection.

The majority of women were non-Hispanic and white with a median age of 35.8 years (Table 1). Among these, $59(89 \%)$ had symptomatic COVID-19. Only $6(9.1 \%)$ were hospitalized, and of these, two developed respiratory failure and required extracorporeal membranous oxygenation (ECMO) support. Two-thirds (66.7\%) of the women who were hospitalized were pregnant at the time of their SARS-CoV-2 infection and hospitalizations. The two women with severe disease requiring ECMO ultimately delivered their infants while receiving treatment.

SARS-CoV-2 viral RNA was detectable in one milk sample each from 7 of the 66 with confirmed infection (Fig. 1). The women with detectable SARS-CoV-2 RNA in their breast milk did not differ in age, race or ethnicity, or other demographic parameters from women with negative breast milk samples (Table 1). For the seven women whose milk samples contained viral RNA, SARS-CoV-2 RNA was not detected in the next specimen of milk available, ranging from 1 to 97 days later (Table 2 and Fig. 1). There was no clinical evidence of infection among any of the infants being breastfed by the seven women with documented SARS-CoV-2 RNA in breast milk.

\section{Cultures and subgenomic RT-PCR reveal no evidence of infectious SARS-CoV-2 in breast milk}

None of the viral cultures of 160 breast milk specimens was positive. We used a second real-time RT-PCR assay to further analyze the seven milk specimens found to contain vRNA for the presence of subgenomic RNA (sgRNA) (Table 2). sgRNAs detected by this assay are spliced RNAs that are produced during coronavirus replication. ${ }^{15}$ The milk specimens were clarified by centrifugation and RNA was extracted from the skimmed milk as before. sgRNA was not detected in any of these seven specimens.

\section{Stability of SARS-CoV-2 in human milk}

We considered the possibility that freezing and thawing of breast milk inactivates SARS-CoV-2 and prevents its detection by culture. $\mathrm{We}$, therefore, added a small inoculum (100 TCID 50 of infectious SARS-CoC-2 to milk samples from two healthy women, performed serial freeze-thaw cycles, and then stored the samples at $4{ }^{\circ} \mathrm{C}$ for 3 days. After this prolonged storage, we added the milk to viral cultures. After 4 days of culture, we removed culture supernatants and used RT-PCR to confirm the presence of viral RNA. Samples that had undergone as many as three freeze and thaw cycles continued to exhibit high infectivity: cytopathic effect spread throughout the cultures within 3 days (just as quickly as positive control wells inoculated with fresh SARS-CoV-2 alone). These experiments demonstrated that the temperature excursions that frequently occur during the handling of breast milk would not prevent us from detecting infectious SARS-CoV-2.

\section{DISCUSSION}

Breast milk is an invaluable source of nutrition to infants ${ }^{16,17}$ and contains numerous antimicrobial factors, including neutralizing antibodies to viruses. ${ }^{18,19}$ Of note, antibodies to SARS-CoV-2 have been detected in the breast milk of recently infected lactating women. ${ }^{20-22}$ However, breastfeeding is clearly a route by which either HIV or HTLV (human T cell lymphotropic virus) can be transmitted to infants. In contrast to these pathogens, hepatitis $B$ virus and hepatitis $C$ virus infections produce chronic infections in women and yet are not contraindications to breastfeeding. ${ }^{23}$

A few small case reports have described the detection of SARSCoV-2 RNA in breast milk 5,24 and one report suggested the possibility of vertical transmission, although contamination could not be ruled out as the source. ${ }^{25}$ Prior reports have generally examined the breast milk of infected mothers for the presence of the virus using RT-PCR methods., ${ }^{925-33}$ In one case, SARS-CoV-2 was said to be detectable by RT-PCR in four milk samples collected between 6 and 10 days after the mother's first positive test. The authors indicated there was no evidence that contamination of the milk samples could have been the source of the virus. However, these reports do not give an overall estimate of the frequency of viral RNA and infectious SARS-CoV-2 in human milk, and the report of possible transmission did not exclude the possibility of contamination of milk occurring at the time of collection. ${ }^{24}$ The rationale for this study was to determine how often SARS- CoV-2 viral RNA was present in breast milk samples and to examine the risk of infection to infants through breast milk. This study is unprecedented in the use of viral cultures to examine a very large number of breast milk specimens, a limitation of prior studies cited by Lackey et al. ${ }^{24}$

We found that SARS-CoV-2 RNA is seldom detected in breast milk samples from women with confirmed SARS-CoV-2 infection. Moreover, our longitudinal follow-up indicates that even when it is detected, it is an unlikely source of infection for the breastfed 
1144

baby: viral RNA was only transiently present and we were unable to culture SARS-CoV-2 from any sample.

This study had several limitations as well as strengths. The collection of breast milk samples was not directly observed and we relied on the maternal report of SARS-CoV-2 test results, symptoms, and treatments received. However, all participants completed a semistructured interview guided by trained study staff who prompted for specifics with the aid of a calendar. In addition, to our knowledge, this study represents the largest number of breast milk samples analyzed to date from women with recent SARS-CoV-2 infection. Nonetheless, even this size may have been too small to permit the identification of factors that would predict the presence of SARS-CoV-2 RNA in breast milk.

We demonstrated that the SARS-CoV-2 maintains its infectivity despite repeated freezing and thawing and storage at $4{ }^{\circ} \mathrm{C}$. While sgRNA (an indicator of virus replication) was not detected in any of the milk specimens already known to contain SARS-CoV-2 RNA, this assay is only moderately sensitive compared to PCR detection of genomic RNA. For example, sgRNA is present in only about half of nasopharyngeal specimens with positive viral cultures (see refs. ${ }^{13,15}$ and unpublished data by P.K.).

\section{CONCLUSION}

These data provide substantial evidence that breastfeeding from women proven or suspected to have had SARS-CoV-2 infection does not represent a hazard for infants.

\section{REFERENCES}

1. World Health Organization. WHO coronavirus disease (COVID-19) dashboard. https://covid19.who.int/ (2021).

2. Bhuiyan, M. U. et al. Epidemiology of COVID-19 infection in young children under five years: a systematic review and meta-analysis. Vaccine 39, 667-677 (2021).

3. Centers for Disease Control and Prevention. Demographic trends of COVID-19 cases and deaths in the US reported to CDC. COVID.CDC.gov (2021).

4. Kaushik, A., Gupta, S., Sood, M., Sharma, S. \& Verma, S. A systematic review of multisystem inflammatory syndrome in children associated with SARS-CoV-2 infection. Pediatr. Infect. Dis. J. 39, e340-e346 (2020).

5. Vardhelli, V., Pandita, A., Pillai, A. \& Badatya, S. K. Perinatal COVID-19: review of current evidence and practical approach towards prevention and management. Eur. J. Pediatr. https://doi.org/10.1007/s00431-020-03866-3 (2020).

6. Kumar, J., Meena, J., Yadav, A. \& Kumar, P. SARS-CoV-2 detection in human milk: a systematic review. J. Matern. Fetal Neonatal Med. https://doi.org/10.1080/ 14767058.2021.1882984, 1-8 (2021).

7. World Health Organization. Breastfeeding and COVID-19. https://www.who.int/ news-room/commentaries/detail/breastfeeding-and-covid-19 (2020).

8. Yeo, K. T. et al. Review of guidelines and recommendations from 17 countries highlights the challenges that clinicians face caring for neonates born to mothers with COVID-19. Acta Paediatr. 109, 2192-2207 (2020).

9. Chambers, C. et al. Evaluation for SARS-CoV-2 in breast milk from 18 infected women. JAMA 324, 1347-1348 (2020).

10. Conzelmann, C. et al. Pasteurization inactivates SARS-CoV-2-spiked breast milk. Pediatrics 147, https://doi.org/10.1542/peds.2020-031690 (2021).

11. Unger, $\mathrm{S}$. et al. Holder pasteurization of donated human milk is effective in inactivating SARS-CoV-2. CMAJ 192, E871-E874 (2020).

12. Walker, G. J. et al. SARS-CoV-2 in human milk is inactivated by Holder pasteurisation but not cold storage. J. Paediatr. Child Health 56, 1872-1874 (2020).

13. Perera, R. et al. SARS-CoV-2 virus culture and subgenomic RNA for respiratory specimens from patients with mild coronavirus disease. Emerg. Infect. Dis. 26, 2701-2704 (2020)

14. Rodriguez-Grande, C. et al. Inference of active viral replication in cases with sustained positive RT-PCRs for SARS-CoV-2. J. Clin. Microbiol. https://doi.org/ 10.1128/JCM.02277-20 (2020).

15. Wolfel, R. et al. Virological assessment of hospitalized patients with COVID-2019. Nature 581, 465-469 (2020).

16. Bode, L., Raman, A. S., Murch, S. H., Rollins, N. C. \& Gordon, J. I. Understanding the mother-breastmilk-infant "triad". Science 367, 1070-1072 (2020).

17. Victora, C. G. et al. Breastfeeding in the 21 st century: epidemiology, mechanisms, and lifelong effect. Lancet 387, 475-490 (2016).
18. Hurley, W. L. \& Theil, P. K. Perspectives on immunoglobulins in colostrum and milk. Nutrients 3, 442-474 (2011).

19. Van de Perre, P. Transfer of antibody via mother's milk. Vaccine 21, 3374-3376 (2003).

20. Fox, A. et al. Robust and specific secretory IgA against SARS-CoV-2 detected in human milk. iScience 23, 101735 (2020).

21. Gao, X. et al. Clinical and immunologic features among COVID-19-affected mother-infant pairs: antibodies to SARS-CoV-2 detected in breast milk. $N$. Microbes N. Infect. 37, 100752 (2020).

22. Pace, R. M. et al. Characterization of SARS-CoV-2 RNA, antibodies, and neutralizing capacity in milk produced by women with COVID-19. mBio 12, https://doi.org/ 10.1128/mBio.03192-20 (2021).

23. American Academy of Pediatrics. In Report from the Committee on Infectious Diseases (eds Kimberlin, D. W., Barnett, E. D., Lynfield, R. \& Sawyer, M.) (American Academy of Pediatrics, 2021).

24. Lackey KA et al. SARS-CoV-2 and human milk: What is the evidence?. Matern Child Nutr. 16, e13032. https://doi.org/10.1111/mcn.13032 (2020).

25. Wu, Y. et al. Coronavirus disease 2019 among pregnant Chinese women: case series data on the safety of vaginal birth and breastfeeding. BJOG 127, 1109-1115 (2020)

26. Bertino, E. et al. Detection of SARS-CoV-2 in milk from COVID-19 positive mothers and follow-up of their infants. Front. Pediatr. 8, 597699 (2020).

27. Carosso, A. R., Cosma, S. \& Benedetto, C. Vaginal delivery in COVID-19 pregnant women: anorectum as a potential alternative route of SARS-CoV-2 transmission. Am. J. Obstet. Gynecol. 223, 612 (2020).

28. Chen, $\mathrm{H}$. et al. Clinical characteristics and intrauterine vertical transmission potential of COVID-19 infection in nine pregnant women: a retrospective review of medical records. Lancet 395, 809-815 (2020).

29. Costa, S. et al. Excretion of SARS-CoV-2 in human breast milk. Clin. Microbiol. Infect. 26, 1430-1432 (2020).

30. Groß, R. et al. Detection of SARS-CoV-2 in human breastmilk. Lancet 395, 1757-1758 (2020).

31. Hinojosa-Velasco, A. et al. A case report of newborn infant with severe COVID-19 in Mexico: detection of SARS-CoV-2 in human breast milk and stool. Int. J. Infect. Dis. 100, 21-24 (2020).

32. Liu, W. et al. Clinical analysis of neonates born to mothers with or without COVID19: a retrospective analysis of 48 cases from two neonatal intensive care units in Hubei Province. Am. J. Perinatol. 37, 1317-1323 (2020).

33. Tam, P. C. K. et al. Detectable severe acute respiratory syndrome coronavirus 2 (SARS-CoV-2) in human breast milk of a mildly symptomatic patient with coronavirus disease 2019 (COVID-19). Clin. Infect. Dis. https://doi.org/10.1093/cid/ ciaa673 (2020).

\section{ACKNOWLEDGEMENTS}

The authors gratefully acknowledge the participation of the women and their infants described in this study.

\section{AUTHOR CONTRIBUTIONS}

P.K. participated in study design, developed the methods for and performed breast milk coronavirus culture experiments under Biosafety Level 3 conditions, and drafted the manuscript. G.M.A. and L.B. were leaders in the design of the study and all aspects of data review, provided financial support, and were integrally involved in manuscript finalization. C.D.C. and K.B. participated in study design, supervised the collection of specimens used in the study, performed the demographic and statistical analysis, and were involved in preparing the manuscript. D.C. was involved in study design, devised and optimized the breast milk quantitative RT-PCR assay, and performed most of the quantitative PCR studies. H.N. prepared the virus stocks used in key experiments and performed breast milk viral cultures under Biosafety level 3 conditions, drafted portions of the "Materials and methods" section, and N.T. aided in designing the study, supervised study organization and sample sharing, performed quantitative RT-PCR assays, and was involved in editing the final manuscript. All authors approved the final manuscript as submitted and agree to be accountable for all aspects of the work.

\section{FUNDING}

P.K. received support from the University of California Los Angeles (UCLA) AIDS Institute, UCLA CFAR (AI028697), the James B. Pendleton Charitable Trust, and the McCarthy Family Foundation. C.D.C. received an award for this study from the University of California Office of the President Emergency COVID-19 Research 
Program, and also received resources for the Human Milk Research Biorepository from the Altman Clinical Translational Research Institute (ACTRI) at UC San Diego funded by the National Institutes of Health $(\mathrm{NIH})$ National Center for Advancing Translational Sciences (NCATS) under Award Number UL1TR001442. L.B. is the UC San Diego Chair of Collaborative Human Milk Research, endowed by the Family LarssonRosenquist Foundation that also provided an unrestricted COVID-19 emergency gift fund. G.M.A. is supported by the IMPAACT Network. Overall support for the International Maternal Pediatric Adolescent AIDS Clinical Trials Group (IMPAACT) was provided by the National Institute of Allergy and Infectious Diseases (NIAID) of the National Institutes of Health (NIH) under Award Numbers UM1Al068632 (IMPAACT LOC), UM1Al068616 (IMPAACT SDMC) and UM1Al106716 (IMPAACT LC), with cofunding from the Eunice Kennedy Shriver National Institute of Child Health and Human Development (NICHD) and the National Institute of Mental Health (NIMH). The content is solely the responsibility of the authors and does not necessarily represent the official views of the $\mathrm{NIH}$

\section{COMPETING INTERESTS}

L.B. reported serving as the UC San Diego Chair of Collaborative Human Milk Research, which is endowed by the Family Larsson-Rosenquist Foundation. Medela Corporation provided milk sample collection materials for this study. C.D.C. reports that shipping of milk samples was financially supported by the Mothers' Milk Bank at Austin, an accredited milk bank and member of the Human Milk Banking Association of North America.

\section{ETHICS APPROVAL AND CONSENT TO PARTICIPATE}

The University of California San Diego Institutional Review Board approved the study, and women provided oral and written informed consent.

\section{ADDITIONAL INFORMATION}

Supplementary information The online version contains supplementary material available at https://doi.org/10.1038/s41390-021-01902-y.

Correspondence and requests for materials should be addressed to Paul Krogstad.

Reprints and permission information is available at http://www.nature.com/ reprints

Publisher's note Springer Nature remains neutral with regard to jurisdictional claims in published maps and institutional affiliations.

Open Access This article is licensed under a Creative Commons Attribution 4.0 International License, which permits use, sharing, adaptation, distribution and reproduction in any medium or format, as long as you give appropriate credit to the original author(s) and the source, provide a link to the Creative Commons license, and indicate if changes were made. The images or other third party material in this article are included in the article's Creative Commons license, unless indicated otherwise in a credit line to the material. If material is not included in the article's Creative Commons license and your intended use is not permitted by statutory regulation or exceeds the permitted use, you will need to obtain permission directly from the copyright holder. To view a copy of this license, visit http://creativecommons. org/licenses/by/4.0/.

(c) The Author(s) 2021 